\section{Photoepilation and skin photorejuvenation: an update}

\author{
Alessandro Martella, ${ }^{1}$ Mauro Raichi ${ }^{2}$ \\ ${ }^{1}$ Former Senior Consultant in \\ Dermatology, University of Modena and \\ Reggio Emilia Medical School, Tiggiano \\ (LE); ${ }^{2}$ Clinical Pharmacology and \\ Biophysics Consultant, Milan, Italy
}

\begin{abstract}
The effectiveness of intense pulsed light (IPL) and laser devices is widely accepted in aesthetic dermatology for unwanted hair removal and treatment of a variety of cutaneous conditions. Overall, most comparative trials have demonstrated similar effectiveness for IPL and laser devices. Literature studies alternatively favor the IPL and laser concepts, but the incidence of severe local pain and side effects were generally lower with IPL. IPL phototherapy, already established as a sound option in photoepilation and treatment of photoaging, hyperpigmentation and other skin conditions, is also considered first choice in the phototherapy of skin vascular malformations. When treating large areas, as often required in photoepilation and many aesthetic dermatology indications, IPL technologies show advantages over laser-based devices because of their high skin coverage rate. Compared to lasers, the wide range of selectable treatment settings, though a strong advantage of IPL, may also imply some more risk of local thermal side effects, but almost only in the hands of poorly trained operators. Overall, the strongest advantages of the IPL technologies are robust technology, versatility, lower purchase price, and the negligible risk of serious adverse effects in the hands of skilled and experienced operators.
\end{abstract}

\section{How the photoepilation story began}

Two landmark dates mark the history of photoepilation and, generally, the application of pulsed light sources in dermatology and aesthetic dermatology.

The introduction of the selective photothermolysis concept in 1984 was the first landmark event. It was a crucial step that deserved a Science paper and opened the way to major advances in photoepilation technology over the past quarter of a century. In the words of the authors, selective damage to pigmented structures, cells, and organelles in vivo with suitably brief pulses of selectively absorbed radiation overcomes the need of precise aiming because inherent optical and thermal properties provide target selectivity. ${ }^{1}$

Proper selection of wavelength in the recommended visible to near-infrared region up to about 1100-1200 nanometers (nm), pulse duration, and the energy density administered over the exposure time or fluence are the three paramount parameters. Compromise among these three parameters is crucial to ensure deep dermal penetration, maximum thermal damage to the melanin chromophore, and minimum absorption by oxyhemoglobin and water. ${ }^{2,3}$

The second landmark date was 1996. In that year the American Food and Drug Administration approved the first ruby-laser device for hair removal. It was so feasible to overcome at least the most severe risks of scarring or hyperpigmentation inherent to electrolysis, performed since 1875 and the only other technology then available for long-term destruction of follicles. ${ }^{4}$ Today light-based hair removal technologies are based either on narrow-waveband lasers or high-intensity incoherent and multi-chromatic pulsed light (intense pulsed light, IPL). Long-term unwanted hair removal has become one of the fastest growing, nonsurgical aesthetic procedures in Europe and all over the world. ${ }^{2}$ Specifically as regards broad-spectrum IPL epilation based on noncoherent 590-1200 nm light sources - typically xenon flash lamps emitting energy in short bursts and operated with cut-off filters - already in 2014 it had become the seventh most popular procedure among plastic surgeons and related specialists, according to the American Society for Aesthetic Plastic Surgery. ${ }^{5}$

\section{The relative merits and liabilities of intense pulsed light and laser systems}

Any chromophore-based photoepilation technology should be an efficient compromise between dermal penetration and targeting of the endogenous melanin in the hair shaft, the outer sheath of the hair follicle infundibulum and the matrix areas (Figure 1). The hair bulb and the germinal matrix are rich in amelanotic stem cells that are most efficiently targeted during the anagen phase of development, when the nonpigmented germinal matrix is closest to pigmented structures. ${ }^{2,6-8}$ IPL and laser devices that operate in the red or near-infrared wavelength region like the long-pulsed 755-
Correspondence: Mauro Raichi,

E-mail: mraichi@gmail.com

Key words: Photoepilation; photorejuvenation; intense pulsed light; dermal remodeling.

Sponsor: Novavision Group S.p.A., Misinto (MB), Italy.

Conflict of interest: the authors declare no potential conflict of interest.

Received for publication: 6 March 2017. Accepted for publication: 2 May 2017.

This work is licensed under a Creative Commons Attribution-NonCommercial 4.0 International License (CC BY-NC 4.0).

(C) Copyright A. Martella and M. Raichi 2017 Licensee PAGEPress, Italy

Dermatology Reports 2017; 9:7116

doi:10.4081/dr.2017.7116

$\mathrm{nm}$ alexandrite laser, the long-pulsed 800 , $810-\mathrm{nm}$ diode laser and the long-pulsed 1064-nm neodymium:yttrium-aluminiumgarnet (Nd:YAG) laser allow for the overall best compromise. ${ }^{6,9}$

The Nd:YAG laser, less effective in light-skinned subjects, could find a somewhat limited but elective niche in dark-skin individuals (Fitzpatrick skin type IV-VI), especially with pseudofolliculitis barbae. ${ }^{8,10}$ The Nd:YAG has the least overall hair removal efficiency while the alexandrite and diode lasers are the best for extensive use. However, post-laser pigmentation may be troublesome with alexandrite devices whilst the need for high levels of fluence increase the risk of complications with diode systems in darker skin types. ${ }^{11}$

IPL systems are possibly more technique-dependent to limit the risk of local pain and irritation. However, they offer the benefit to be highly versatile due to the wide range of emitted wavelengths when hair or skin colors are not ideal for laser photoepilation. ${ }^{8}$ The hair removal benefits of IPL technologies are most significant in individuals with dark hair and light skin, a common Caucasian phenotype and the dominant phenotype in East Asia. Hair that is growing after epilation is also thinner and lighter in color, and thus often another ideal candidate for IPL re-treatment several months after the first IPL or laser procedure. ${ }^{8}$ A decade ago, there had been some isolated criticism of IPL, related to the need for early IPL devices to be very highly powered to destroy hair. ${ }^{12}$ Yet, a decade ago is much time in this rapidly evolving field. Some recent intra-patient-controlled split- 
face comparisons seem to indicate that IPL could offer some benefits compared with laser devices in terms of hair reduction, patient satisfaction, and local pain. A 2013 left-to-right split-face assessor-blinded study compared a IPL $\left(\lambda_{\mathrm{em}}=600-950 \mathrm{~nm}\right)$ and a diode laser device for removal of unwanted axillary hair (6 sessions at 4week intervals in 30 subjects with skin type II-III; hair counts assessed with close-up photographs). ${ }^{13}$ The 3- and 12-month mean hair reduction was slightly higher with the diode laser system $(59.7 \%$ vs $42.4 \%$ and $69.2 \%$ vs $52.7 \%$ from baseline for laser and IPL treatments, respectively), but at significantly higher pain cost compared with the almost pain-free IPL technology $(3.7 \pm 2.1 \mathrm{vs}$ $1.6 \pm 1.4$, respectively; $10-\mathrm{cm}$ visual analogue scale). ${ }^{13}$ Similar results were seen in a double-blind, intra-patient-controlled randomized study of axillary hair removal in 21 women after application (6 sessions) of a diode laser on one axilla and IPL on the other one. The number of hair shafts was lastingly and similarly reduced with both hair reduction systems, but local pain was significantly more disturbing with the diode laser. $^{14}$

\section{Ultra-refining the chromophore targeting process}

Some advanced IPL systems are being introduced that allow to concentrate almost all emitted energy in very narrow wavelengths selected within the ample waveband of IPL xenon flash lamps. For instance, an advanced Italian IPL-derived technology [IFL ${ }^{\text {TM }}$ (Incoherent Fast Light), Novavision Group S.p.A., 20826 Misinto (MB), Italy], when used in photoepilation mode at up to 3.3 light pulses per second, allows an extremely high selection of the wide xenon lamp waveband by focusing the energy emission on 700,810 and $890 \mathrm{~nm}$ wavelengths. Ultraviolet emissions are preliminary filtered away by default by the ultratransparent cerium-supplemented borosilicate glass of the IFL TM light emitter acting together with a permanently installed 420$\mathrm{nm}$ cut-off filter. The local safety allowed by wavelength selection is further improved by avoiding all contacts of the device with the skin. At the same time, the technological improvements of the system allow to increase the number of spots up to 210,000 .

Summarizing, the photoepilation story that began in 1984 with a landmark Science paper is still going on and technology is still making strong headways; and not only in photoepilation.

\section{The expanding scope for intense pulsed light beyond photoepilation}

In 1990, a high-intensity flash lamp was first described as a new tool for treating vascular lesions; the first IPL medical device aimed to benign vascular lesions was commercially launched in $1994 . .^{15}$
Since those years, many features of first-generation IPL devices have undergone technological refinements, with special reference to the xenon gas-discharge flash lamp and the electronic control of the capacitor banks. Such capacitors store and discharge the electrical energy that generates the pulsed polychromatic high-intensity light. The computer-assisted selection of

A

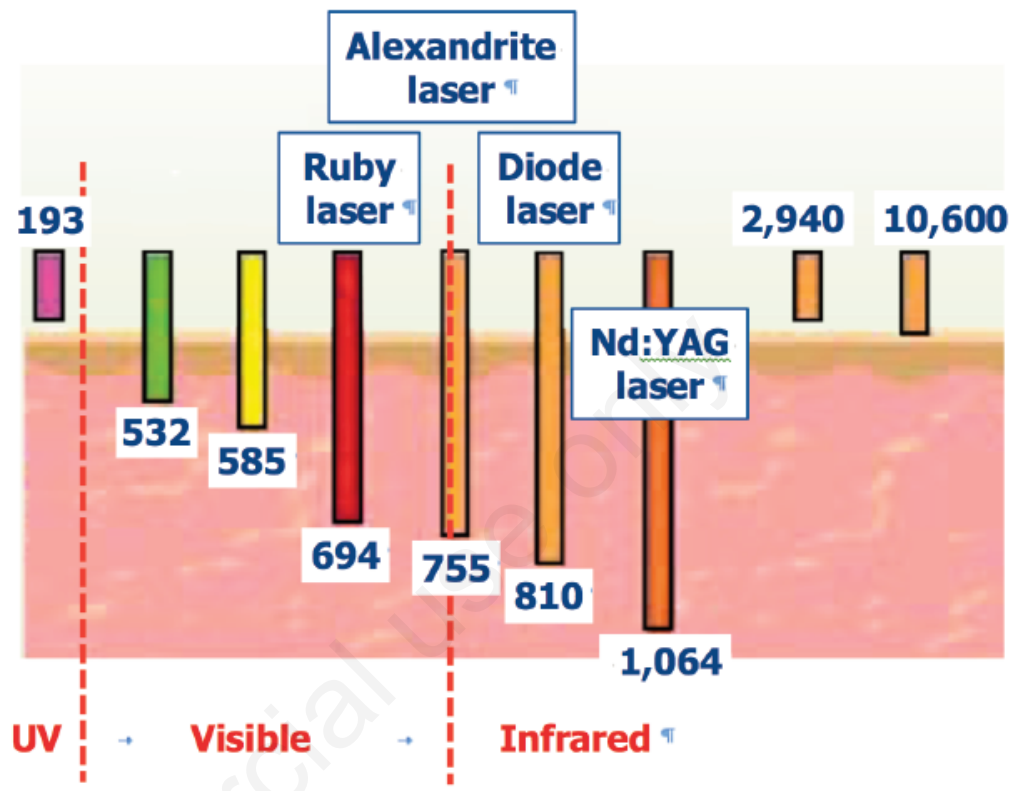

IPL and laser wavelength (nm)

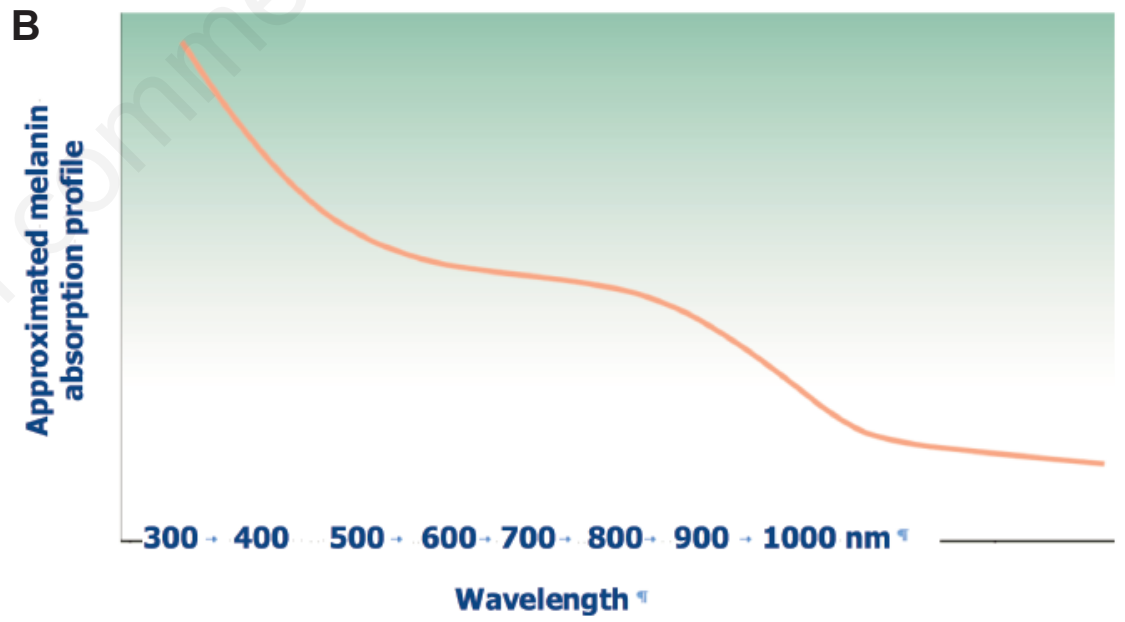

Figure 1. A) Relationship between intense pulsed light (IPL) and laser wavelength and depth of penetration into the epidermis and dermis. At wavelengths within the low absorption index of water (400 to $1000 \mathrm{~nm}$, visible and near-infrared spectrum), dispersal within the dermis decreases with increasing wavelengths whilst penetration deepens and photothermolytic effects become stronger. The IPL wavelength window is about 590 to $1,200 \mathrm{~nm}$. The 694-nm ruby laser is no longer used in photoepilation because of strong absorption by melanin and high risk of adverse effects in tanned and darker skin types and hypopigmented areas observed even in skin type II. Nd:YAG: neodymium:yttriumaluminium-garnet. $\left.{ }^{6,1} 1 \mathrm{~B}\right)$ Diagram illustrating the overall relationship between wavelengths in the visible and near-infrared spectrum and electromagnetic energy absorption by melanin. Energy absorption by the chromophore and thermal biological effects are high in the low visible spectrum and decrease with increasing wavelengths. In spite of high dermal penetration, wavelengths higher than 1,100 nanometers $(\mathrm{nm})$ are less useful for selective photothermolysis. ${ }^{8}$ 
treatment settings is also being steadily improved and water filters have been developed to absorb most infrared radiation. The IFL $^{\mathrm{TM}}$ technology previously discussed is an example of such engineering improvements.

Beyond photoepilation, the versatile range of IPL wavelengths (Figure 2) has been applied to a wide spectrum of dermatological conditions, such as photodamaged skin, acne vulgaris, epidermal and dermal pigmented and vascular lesions, angiokeratoma, and hypertrophic scars and keloids. Available evidences suggest IPL might be the treatment of choice in at least some of such cutaneous disorders. ${ }^{16,17}$

The range of cutaneous disorders amenable to IPL treatment continues to widen. A recent evidence-based set of recommendations, based on the highest level of evidence available to guide physicians in the IPL treatment of dermatologic diseases, found Level 1 evidence for melasma, acne vulgaris, and telangiectasia; Level 2 evidence for lentiginous disease, acne rosacea, capillary malformations, actinic keratoses, and sebaceous gland hyperplasia; Level 3 or lower evidence for poikiloderma of Civatte, venous malformations, infantile hemangiomas, hypertrophic scars and keloids, superficial basal cell carcinoma, and Bowen's disease. ${ }^{18}$ Other dermatological disorders have also been treated with IPL with satisfactory results: port-wine stains, a common congenital vascular malformation occurring in up to $25 \%$ of individuals since infancy, Becker's nevus, a male-predominant benign hypermelanotic birthmark on the shoulders, chest or lower back, and pilonidal or sacro-coccygeal cysts containing hair and skin debris. ${ }^{19}$ Disseminated porokeratosis, presenting as asymptomatic or mildly itching atrophic plaques surrounded by an hyperkeratotic border histologically known as cornoid lamella, and the soft warty or squamous crusted, yellow-brown surface of seborrheic keratosis have also been treated quite successfully with IPL. ${ }^{19}$

\section{Biological mechanisms of intense pulsed light phototherapy and photorejuvenation}

Findings in photoaging due to chronic exposure to UV sunlight include wrinkling and rough skin texture, altered pigmentation and loss of elasticity. Even with first-generation IPL devices, improved texture and telangiectasias were reported 4 years after IPL treatment (median, 3 sessions) by, respectively, $83 \%$ and $82 \%$ of individuals with skin types I-IV; improvements in mot- tled pigmentation persisted in $79 \%$ of treated subjects. ${ }^{20}$ As regards the biological mechanisms underlying IPL photorejuvenation, epithelial and dermal structures appear to be preserved in the weeks following the IPL procedure with no further lesion to the sun-damaged skin. ${ }^{21}$ Cosmetic benefits are thus most probably unrelated to destruction of pre-existing dermal structures. Conversely, increased collagen deposition in the upper papillary and upper reticular dermis and a more neat arrangement of elastin fibers have long been known to have a role in the clinical and aesthetic improvements. ${ }^{21,22}$

Dermal dendritic cells are likely to be the biological target in IPL photorejuvenation, as suggested by the expression of the heat-induced protein hsp70 (Heat Shock Protein 70 kilodaltons) and procollagen 1 by these cells as markers of their activation. Activation of dermal dendritic cells might be the underlying event that ultimately leads to collagen deposition by dermal fibroblasts. ${ }^{21}$ Advanced IPL developments are being devised to activate such cellular photorejuvenation mechanisms. A most recent example is the PhotoActivating System (PhAS ${ }^{\mathrm{TM}}$ ) program of the IFL ${ }^{\mathrm{TM}}$

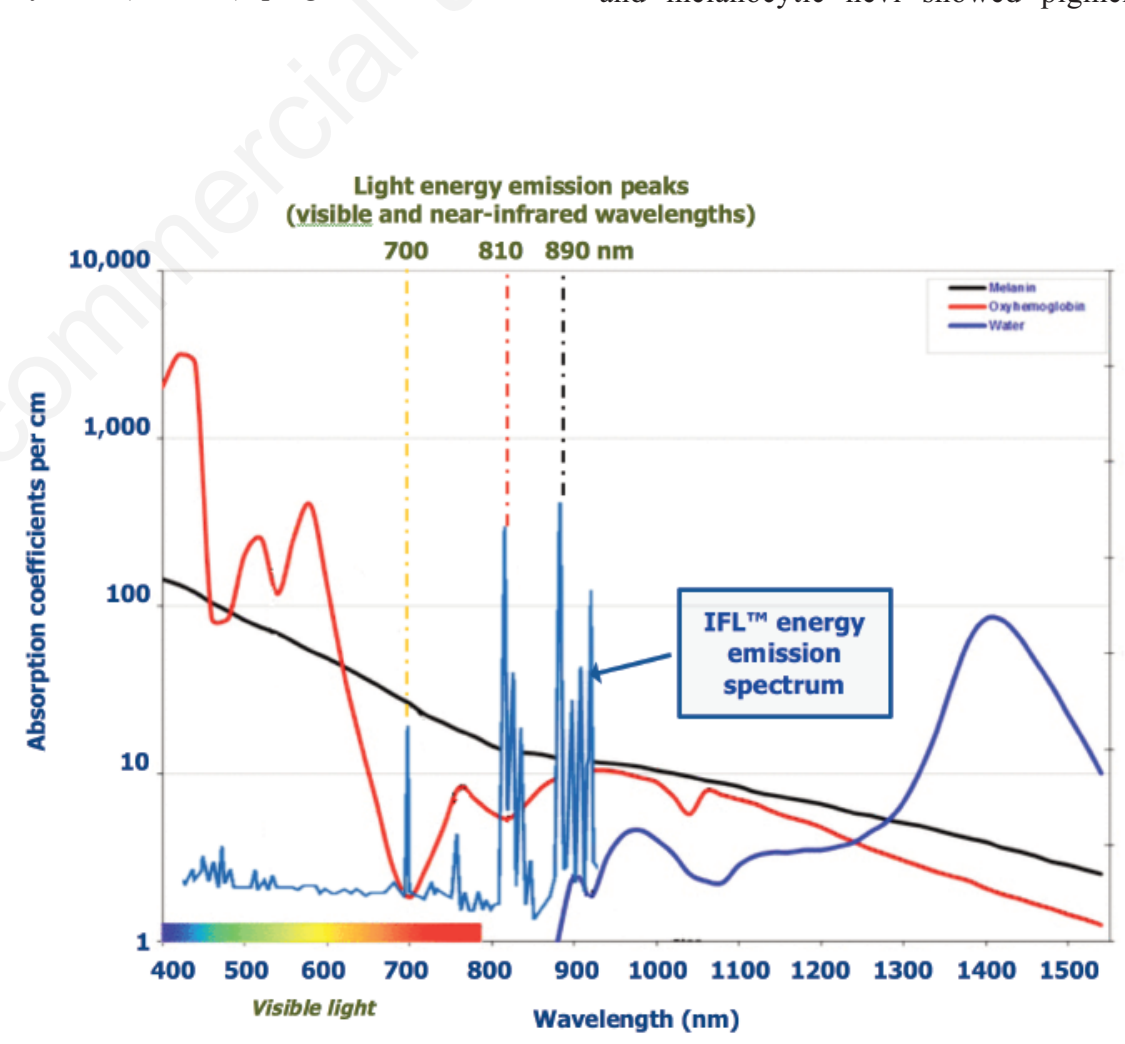

Figure 2. Light absorption spectra of skin chromophores (melanin and oxyhemoglobin) and water in visible and infrared wavelengths with evidence of the strongest energy emission peaks of IPL flash-lamp devices within their operating visible and near-infrared wavelengths. In advanced IPL devices like Incoherent Fast Light ${ }^{\mathrm{TM}}$ (IFL ${ }^{\mathrm{TM}}$ ), the emitted light energy is selectively concentrated within these energy-emission peaks whilst the ultra-transparent low-residue water in the closed-loop cooling system extensively filters away infrared wavelengths longer than 900 nanometers. 
reduction with an average clearance of $74.2 \%$ and $66.3 \%$ of lesions, respectively. Superficial crusting and ulceration (average diameter on day $5,3.7 \mathrm{~mm}$ ) followed by some degree of erythema were observed in most treated lesions, but healing was complete within 30 days. ${ }^{24}$ Figure 3 illustrates the IPL and IFL ${ }^{\mathrm{TM}}$ wavelength range ideally suited to phototherapy of cutaneous dyschromia.

Skin vascular lesions such as facial telangiectasia and port-wine stains have traditionally been treated with the pulsed dye laser. Such lesions are another indications where IPL is at least as effective as the gold standard. IPL may even be considered first choice because it is often successful when the pulsed dye laser fails. The mechanism of action is related to selective absorption of IPL energy by hemoglobin within target blood vessels. ${ }^{25,26}$ In patients with port-wine stains resistant to multiple pulsed dye laser treatments, almost half of the patients (46.7\%) responded to four IPL sessions with a more than $50 \%$ reduction of lesions. The average clearance for the responders was $83.9 \pm 9.5 \%$. Most of such IPL responders $(85.7 \%)$ obtained reductions between $75 \%$ and $100 \%$ of their basal stains; only lesions in the central part of the $\mathrm{V}_{2}$ face dermatome failed to respond. Preference for IPL over multiple pulsed dye laser was expressed by $93.5 \%$ of the patients. ${ }^{26}$ IPL technologies have also been long used in the treatment of acne vulgaris. IPL seems to act by targeting both inflammation and sebaceous glands. With a few treatment sessions, IPL reduces the density of the inflammatory cell infiltrate and the surface area of sebaceous glands, especially in more inflammatory acne variants, in parallel with reduction of the Cardiff Acne Disability Index or other scores of clinical impact. ${ }^{27}$

The molecular mechanism of such powerful anti-inflammatory action could be a novel anti-Tumor Necrosis Factor- $\alpha$ effect independent of interleukin-10 up-regulation. ${ }^{28}$ Selective photothermolysis of blood vessels that supply sebaceous glands could also contribute to the acne-suppressing efficacy by reducing the sebum secretion rate. Such photodynamic effect could be mediated by energy absorption by coproporphyrins produced by Propionibacterium acnes (absorption peaks: 400, 510, 542, $578,630,665 \mathrm{~nm}$ ) with generation of bacteridical reactive oxygen species. ${ }^{29}$

All light-based therapies, including IPL, are especially effective in treating inflammatory acne vulgaris with minimal side effects. This was most recently confirmed in a 2016 single-blind, split-face clinical trial that compared the clinical efficacy of 3 sessions of IPL on the right side of the face and 1,064-nm Nd:YAG laser on the left side at 4-weeks intervals in 74 individuals with mild to severe facial acne. The reduction of inflammatory papules, pustules, nodules

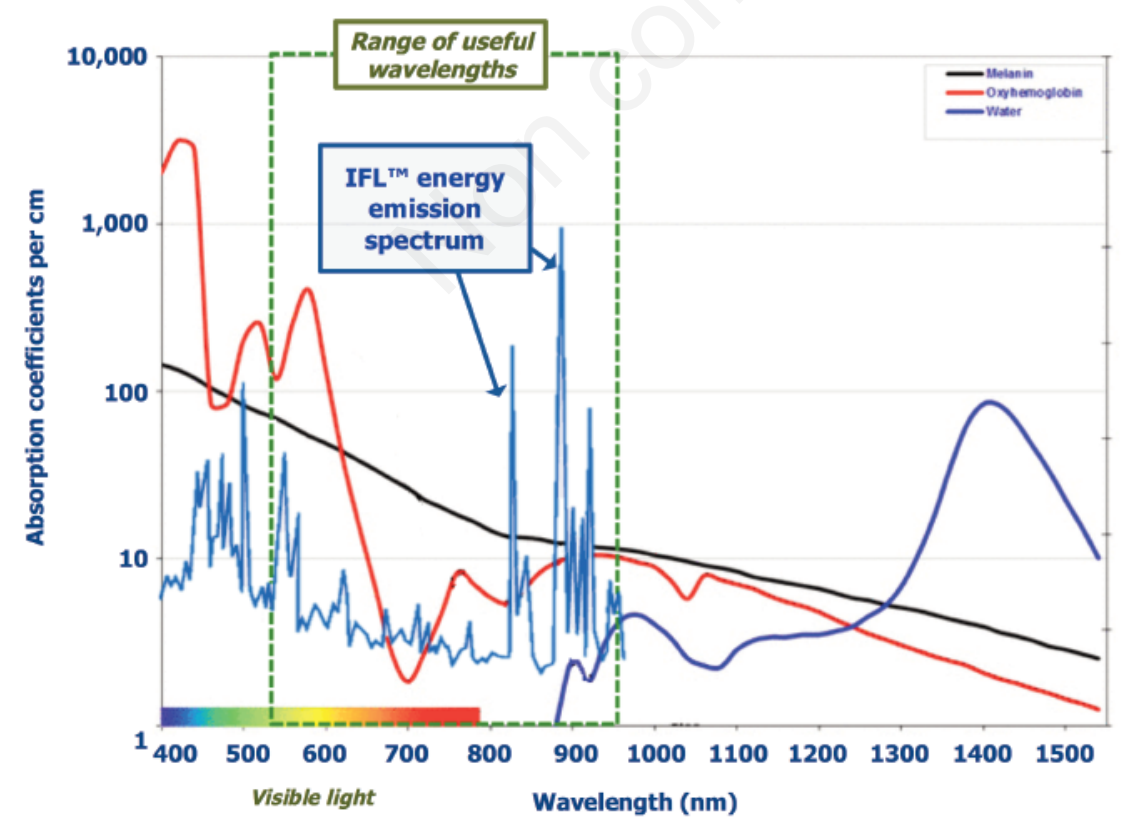

Figure 3. Light absorption spectra of skin chromophores (melanin and oxyhemoglobin) and water in visible and infrared wavelengths with evidence of the useful range of wavelengths in the IPL phototherapy of disorders of cutaneous pigmentation. and cysts was similar with both phototherapy strategies $(-67.1 \%$ and $-70.2 \%$, respectively, with IPL and Nd:YAG laser) while response of non-inflammatory comedones was equally modest $(-18.3 \%$ and $-19.3 \%$, respectively). ${ }^{30}$

\section{Summarizing}

Versatility in treating many dermatological conditions as well as lower commercial costs and more robust technology are strong advantages for IPL technologies compared to laser devices. This may be true for both photoepilation and treatment of several skin disorders, for which IPL is often the firstchoice option. The large spot sizes allowed by IPL shorten the time needed for photorejuvenation sessions while the troublesome need to apply an optical coupling gel has been eliminated with the most advanced pulsed light devices. ${ }^{29}$

IPL technologies developed over the very last years like IFL ${ }^{\text {TM }}$ have also eliminated the emission of sigmoidal-shaped pulses, another weak point of older IPL devices. Shifts in spectral and fluence distribution within the pulse are unavoidable with non-square-shaped pulses. Thanks to the large capacitor banks in advanced IPL devices, variable current are no longer delivered to the xenon flash lamp and roughly square-shaped pulses are dependably emitted. Some minor difficulties with handling and the weight of the handpiece incorporating both the lamp and the lampcooling system are a little price that has still to be paid for all the benefits of the most advanced IPL technologies. ${ }^{29}$ It does not seem a heavy price to pay.

\section{References}

1. Anderson RR, Parrish JA. Selective photothermolysis: precise microsurgery by selective absorption of pulsed radiation. Science 1983;220:524-7.

2. Haedersdal M, Beerwerth F, Nash JF. Laser and intense pulsed light hair removal technologies: from professional to home use. Br J Dermatol 2011;165:31-6.

3. Dierickx CC. Hair removal by lasers and intense pulsed light sources. Dermatol Clin 2002;20:135-46.

4. Richards RN, Meharg GA. Electrolysis: observations from 13 years and 140,000 hours of experience. J Am Acad Dermatol 1995;33:662-6.

5. DiBernardo BE, Pozner JN. Intense pulsed light therapy for skin rejuvena- 
tion. Clin Plast Surg 2016;43:535-40.

6. Drosner M, Adatto M; European Society for Laser Dermatology. Photoepilation: guidelines for care from the European Society for Laser Dermatology (ESLD). J Cosmet Laser Ther 2005;7:33-8.

7. Serrano-Grau P, Campo-Voegeli A, Romero D. Photodepilation. Actas Dermosifiliogr 2009; 100:351-61.

8. Mandt N, Troilius A, Drosner M. Epilation today: physiology of the hair follicle and clinical photo-epilation. J Investig Dermatol Symp Proc 2005;10:271-4.

9. Casey AS, Goldberg D. Guidelines for laser hair removal. J Cosmet Laser Ther 2008;10:24-33.

10. Schulze R, Meehan KJ, Lopez A, et al. Low-fluence 1,064-nm laser hair reduction for pseudofolliculitis barbae in skin types IV, V, and VI. Dermatol Surg 2009;35:98-107.

11. Sadighha A, Mohaghegh Zahed G. Meta-analysis of hair removal laser trials. Lasers Med Sci 2009;24:21-5.

12. McGill DJ, Hutchison C, McKenzie E, et al. A randomised, split-face comparison of facial hair removal with the alexandrite laser and intense pulsed light system. Lasers Surg Med 2007;39:767-72.

13. Klein A, Steinert S, Baeumler W, et al. Photoepilation with a diode laser vs. intense pulsed light: a randomized, intrapatient left-to-right trial. $\mathrm{Br} \mathrm{J}$ Dermatol 2013;168:1287-93.

14. Ormiga $\mathrm{P}$, Ishida CE, Boechat $\mathrm{A}$, Ramos-E-Silva M. Comparison of the effect of diode laser versus intense pulsed light in axillary hair removal. Dermatol Surg 2014;40:1061-9.

15. Goldman MP. Treatment of benign vascular lesions with the Photoderm VL high-intensity pulsed light source. Adv Dermatol 1997;13:503-21.

16. Erol OO, Gurlek A, Agaoglu G, et al. Treatment of hypertrophic scars and keloids using intense pulsed light (IPL). Aesthetic Plast Surg 2008;32:902-9.

17. Morais P, Santos AL, Baudrier T, et al. Angiokeratomas of Fabry successfully treated with intense pulsed light. J Cosmet Laser Ther 2008;10:218-22.

18. Wat H, Wu DC, Rao J, Goldman MP. Application of intense pulsed light in the treatment of dermatologic disease: a systematic review. Dermatol Surg 2014;40:359-77.

19. Piccolo D, Di Marcantonio D, Crisman $\mathrm{G}$, et al. Unconventional use of intense pulsed light. Biomed Res Int 2014; 2014:618206.

20. Weiss RA, Weiss MA, Beasley KL. Rejuvenation of photoaged skin: 5 years results with intense pulsed light of the face, neck, and chest. Dermatol Surg 2002;28:1115-9.

21. Prieto VG, Diwan AH, Shea CR, et al. Effects of intense pulsed light and the 1,064 nm Nd:YAG laser on sun-damaged human skin: histologic and immunohistochemical analysis. Dermatol Surg 2005;31:522-5.

22. Goldberg DJ. New collagen formation after dermal remodeling with an intense pulsed light source. J Cutan Laser Ther 2000;2:59-61.

23. Galeckas KJ, Collins M, Ross EV, Uebelhoer NS. Split-face treatment of facial dyschromia: pulsed dye laser with a compression handpiece versus intense pulsed light. Dermatol Surg 2008;34:672-80.

24. Bjerring $P$, Christiansen $K$. Intense pulsed light source for treatment of small melanocytic nevi and solar lentigines. J Cutan Laser Ther 2000;2:177-81.

25. Tanghetti EA. Split-face randomized treatment of facial telangiectasia comparing pulsed dye laser and an intense pulsed light handpiece. Lasers Surg Med 2012;44:97-102.

26. Bjerring $\mathrm{P}$, Christiansen K, Troilius A. Intense pulsed light source for the treatment of dye laser resistant port-wine stains. J Cosmet Laser Ther 2003;5:713.

27. Barakat MT, Moftah NH, El Khayyat MA, Abdelhakim ZA. Significant reduction of inflammation and sebaceous glands size in acne vulgaris lesions after intense pulsed light treatment. Dermatol Ther 2017 [Epub ahead of print].

28. Taylor M, Porter R, Gonzalez M. Intense pulsed light may improve inflammatory acne through TNF- $\alpha$ down-regulation. J Cosmet Laser Ther 2014;16:96-103.

29. Babilas P, Schreml S, Szeimies RM, Landthaler M. Intense pulsed light (IPL): a review. Lasers Surg Med 2010;42:93-104.

30. Mohamed EE, Tawfik K, Elsaie M. Intense pulsed light versus 1,064 longpulsed neodymium:yttrium-aluminumgarnet laser in the treatment of facial acne vulgaris. J Clin Diagn Res 2016; 10:WC01-3. 